\title{
La actividad tutorial, un soporte académico definitivo para los estudiantes de fisioterapia, fonoaudiología y terapia ocupacional de la Facultad de Rehabilitación y Desarrollo Humano de la Universidad del Rosario
}

\section{Maria del Carmen Urrea González Fisioterapeuta. \\ Universidad Industrial de Santander \\ Profesora Facultad de Rehabilitación \\ y Desarrolo Humano \\ Coordinadora programa de tutorías. \\ Programa de Fisioterapia \\ Universidad del Rosario. \\ E-mail: caurrea@urosario.edu.co}

Sandra Pulido Sánchez.

Fonoaudiologa.

Universidad Nacional de Colombia

Profesora Facultad de Rehabilitación

y Desarrollo Humano

Especialista en epidemiologia.

Universidad del Rosario

Coordinadora programa de tutorías.

Programa de Fonoaudiología

Universidad del Rosario.

E-mail: spulido@urosario.edu.co

Angélica Maria Monsalve Robayo

Terapeuta Ocupacional. Universidad Colegio

Mayor de Nuestra Señora del Rosario.

Profesora Facultad de Rehabilitación

y Desarrolo Humano.

Master en Gerontologia.

Universidad de Salamanca. España

Coordinadora programa de tutorías.

Programa de Terapia Ocupacional.

Universidad del Rosario.

E-mail: anmonsal@urosario.edu.co

\section{Introducción}

¿Cómo organizo mi plan de estudios? $¿$ Es posible cursar doble programa académico? ¿A quién acudo para aclarar las dudas que tengo en una asignatura aparte de mi profesor? ¿Dónde puedo encontrar información acerca de este tema? ¿Cómo accedo algún servicio específico de la universidad? ¿Tengo un problema personal, a dónde debo acudir? ¿Inscribí menos créditos de los que debia, es posible el próximo periodo adelantar asignaturas? ¿No tengo claridad en algunos aspectos del programa académico que elegí, es posible aclararlos en la tutoria? ¿Qué apoyo me brinda la universidad cuando me encuentro en situación de periodo de prueba o cursando por tercera vez una asignatura? ¿Qué consecuencias trae el perder asignaturas o tener un bajo desempeño académico? ¿Qué orientación recibo antes de culminar mis estudios que me brinde herramientas para el ingreso al mundo laboral? 
Estas son algunas de las preguntas que hacen los estudiantes cuando ingresan, durante y en la finalización de sus estudios universitarios. Responder a estas preguntas desde el programa de tutorias en la Facultad de Rehabilitación y Desarrollo Humano representa no solamente el conocimiento de los planes de estudios de cada programa de la Facultad, de los servicios con que cuenta la Universidad o de las redes de información que apoyan los procesos académicos para la formación integral de nuestros estudiantes, sino también, el acercamiento a su proyecto de vida, a sus intereses y expectativas hacia el futuro.

* Resumen El presente artículo es una reflexión en torno a la tutoría como un servicio educativo que constituye para la Facultad de Rehabilitación y Desarrollo Humano el soporte de desarrollo académico para los estudiantes, teniendo como objetivo primordial brindar atención individual y grupal en la que se ofrece orientación y acompañamiento en su proceso de formación desde dimensiones pedagógica, sociológica y antropológica.

\section{Palabras claves}

Tutoría, estudiante, tutor, dimensión pedagógica, dimensión sociológica y dimensión antropológica.

This article is a reflection around the tutorial as an educative service than is for the Rehabilitation and Human Development Faculty the support of academic development for the students and its objective is to give individual and group attention to offers orientation and accompaniment in their formative process from pedagogic, sociologic and anthropologic dimension.

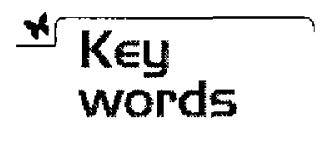

Tutoring, student, tutor, pedagogic dimension, sociologic dimension and anthropologic dimension. 


\section{Las dimensiones de la tutoría}

La actividad tutorial y quienes la desarrollan poseen ciertas características que se fundamentan a partir de dimensiones pedagógica, sociológica y antropológica que serán objeto de estudio del presente artículo, generando en la Facultad oportunidades de aplicación y análisis desde 1999.

\section{Dimesión pedagógica de la tutoría}

Esta concepción lleva necesariamente a reflexionar en torno a la educación como un proceso de socialización que le permite al ser humano desarrollarse dentro de un contexto sociocultural, que implica un sentido transversal de integralidad y pluridimensionalidad y no un simple enfoque instruccional.

Desde esta visión, se considera como un proceso de interacción entre personas con fines posibilitadores de cambio, de transformación, de individualidad, autorrealización y autonomía durante su ciclo vital. (1)

La tutoría desde la dimensión pedagógica y como lo propone Galeano, "responde a un ejercicio didáctico por medio del cual la academia cumple con su objetivo de formar integralmente al alumno dentro de un currículo abierto, flexible y pertinente, para que los estudiantes encuentren en la Universidad la ruta de desarrollo personal y profesional competente y coherente con el ser, el pensar y el hacer". (2)

La Facultad de Rehabilitación y Desarrollo Humano, a través de sus progra- mas de Fisioterapia, Fonoaudiología y Terapia Ocupacional asume esta dimensión propuesta por Galeano, concibiendo la tutoría como el soporte de desarrollo académico y personal que le permite a los estudiantes sentirse parte de la comunidad académica, en donde la gestión de los procesos de seguimiento y apoyo garantizan la autonomía y permanencia en cada uno de los programas, la cual se realiza en modalidades de tutoría individual y grupal, como estrategias metodológicas en donde la pedagogía como reflexión permanente sobre los procesos de enseñanza-aprendizaje, constituyen la forma como se hace operativo el proceso de interacción entre tutor-estudiante-grupo de estudiantes.

Adicionalmente estas modalidades se presentan transversalmente sobre el esquema de desarrollo de la tutoria, el cual es propuesto por la política general a nivel institucional. En el esquema que se describe a continuación se establecen los diferentes servicios y momentos en los que se operacionaliza el programa en la Facultad de Rehabilitación y Desarrollo Humano: 


\section{PRIMER MOMENTO}

\section{Proceso de admisión}

> Participación en la realización de entrevistas

$>$ Identificación de casos que requieren tutoría

\section{SEGUNDO MOMENTO}

\section{Proceso de formación a lo largo de la vida universitaria}
A. Asesoría
B. Orientación
Curricular
C. Apoyo
Integral Medio
D. Apoyo Egreso
Universitario
Universidad

Resuelve inquietudes y dificultades asociadas con el proceso de aprendizaje de los contenidos en asignaturas.
Apoya el proceso de organización del plan de estudios e inscripción de asignaturas, identificación de situaciones problémicas a nivel académico y personal para ser remitidos a otras instancias de apoyo de la Universidad.
Provee un espacio al estudiante para que logre prepararse y adquirir destrezas que le permitan mejorar el desempeño académico y personal durante su vida universitaria.
Orienta aspectos relacionados con el mundo del trabajo y el ingreso a la vida laboral.

\section{Dimensión sociológica de la tutoría}

Esta dimensión hace referencia al valor de lo social y lo colectivo en el mundo universitario y del ejercicio de la tutoría como un componente inherente a la formación, dirigida a impulsar y facilitar el desarrollo integral de los estudiantes desde sus potencialidades intelectuales, afectivas, personales y sociales. (3)

El desarrollo integral por el que la Universidad trabaja dia a día, res- ponde entre otras a una función social que busca satisfacer una necesidad e interés del estudiante, de la familia y de la sociedad, que se circunscriben al proceso educativo como un derecho que se expresa en la libertad y autonomía de ir construyendo un proyecto de vida que aporte de manera significativa al desarrollo del país. 
Nuestra sociedad se organiza alrededor del conocimiento y exige de las personas estar a la vanguardia de los avances tecnológicos, informáticos, científicos, culturales, entre otros, que le permitan reconstruir permanentemente la realidad y operar como mecanismo de control social y de direccionamiento del cambio y de la innovación. Para lograr este propósito la Universidad debe poseer elementos de análisis que favorezcan la orientación asertiva del proceso formativo. (4)
La Universidad del Rosario dentro de su visión plantea garantizar aportes al desarrollo económico, político, social y cultural de la sociedad a través de la formulación de políticas institucionales que posibiliten la formación integral desde una dimensión sociológica tanto en el proceso de aprendizaje como en la interacción de la comunidad académica, visualizando la tutoría como una de las estrategias prioritarias para el desarrollo de la autonomia de los estudiantes y el manejo de competencias con sentido de responsabilidad social. (5)

\section{Dimensión antropológica de la tutoría}

Desde esta dimensión se hace necesario plantear la visión que tenemos de hombre (tutor-estudiante) y las relaciones que se establecen entre ellos durante el proceso tutorial.

El hombre tutor se presenta como un sujeto fundamental en la evolución psicosocial de otro individuo, pues se convierte en herramienta de la interacción social con los demás, con el conocimiento y con la comprensión de sí mismo. Dicha interacción en la acción tutorial requiere que el profesor sea el elemento dinámico que motive al estudiante hacia la búsqueda y organización del conocimiento. El tutor es visto entonces, como componente de la comunidad universitaria que no solo tiene responsabilidad con la ciencia sino con el hombre.

Estos elementos se recogen en un perfil que caracteriza al profesor-tutor como persona flexible, abierta al cambio, capaz de analizar su enseñanza, crítico consigo mismo, amplio en el dominio de destrezas cognitivas y relacionales, capaz de trabajar en equipo y de investigar sobre su propia enseñanza, reflexivo sobre la práctica, con competencias académicas básicas, con destrezas para la toma de decisiones y modelo en la adquisición y uso del conocimiento.

Desde esta perspectiva, el estudiante se concibe como un sujeto activo que se reconoce y se desarrolla a través del mismo proceso de aprendizaje, que incorpora a su proceso formativo el acervo cultural, familiar, social y personal que le permite construir significados y sentidos a partir del saber, el saber hacer y el ser, expuestos en un currículo. Esta interacción permanente entre el contexto educativo y la persona facilita el cambio continuo entre ambos. 
El estudiante se visualiza como un ser humano integrado además por los componentes vocacional, interpersonal e intelectual. El primero hace referencia a sus intereses e inclinaciones hacia la formación en un área específica de conocimiento, producto de la reflexión personal hacia la determinación de un proyecto de vida. El segundo comprende la disposición del estudiante hacia la empatía, la interacción constructiva, armónica y positiva con otras personas en diferentes contextos. El tercero involucra las competencias básicas que le permiten desarrollar la crítica, la reflexión, el análisis, la comunicación, el trabajo en equipo, la toma de decisiones y el uso productivo del conocimiento. (6)

Las individualidades de cada sujeto en este proceso de negociación de significados hace explicito la consideración de que no siempre se debe brindar el mismo tipo de apoyo ni de la misma manera a todos los estudiantes.

La tutoria entonces se asume como un ejercicio de interacción donde la colaboración, la cooperación y la comunicación son elementos sustanciales para la orientación académica, laboral e incluso personal de los estudiantes. Es a través de la interacción comunicativa entre tutor y tutoriado que se aprende a aprender $y$ a desaprender, a través de la comprensión de explicaciones complejas, del entendimiento de conceptos que no se refieren a realidades concretas, de la expresión de significados con precisión, de la formulación de mensajes adecuados y pertinentes, de la argumen- tación con lógica y de la resolución de desacuerdos conceptuales para la construcción de ciudadanía. (7)

Finalmente, la Universidad del Rosario recoge todos estos elementos y concibe la tutoría como "Una estrategia pedagógica y de formación que brinda cada una de las unidades académicas a sus estudiantes, con el fin de apoyarlos y orientarlos en su proceso de formación integral, así como estimular el desarrollo de habilidades para alcanzar una ganancia en el aprendizaje de su disciplina. Por lo tanto está dirigida a potenciar las capacidades de los estudiantes y a superar las dificultades surgidas en los procesos de aprendizaje". (8)

La Facultad de Rehabilitación y Desarrollo Humano acogiéndose a esta concepción de tutoría, tiene en cuenta que la calidad en la formación de fisioterapeutas, fonoaudiólogos y terapeutas ocupacionales se fortalece desde la gestión y actualización de los procesos de evaluación, de las metodologías de enseñanza-aprendizaje, de la relación profesor-estudiante y de la revisión permanente del currículo que garantizan la excelencia personal y profesional de nuestros egresados.

"El conocimiento y la tecnologia son cambiantes, pero la calidad de ciudadano y la actitud hacia el conocimiento son permanentes". (9)

"La tutoría es la protección que favorece el crecimiento de las alas de nuestros pupilos. Una hermosa tarea de preparara otros para su propia libertad." (10) 


\section{Bibliografî́a}

(1) DIAZ, LUZ A., FAJARDo, Adriana.

Modelo pedagógico. Facultad de Rehabilitación y Desarrollo Humano. Univerșidad del Rosario.

Bogotá. 2004

(2) GALEANO, Aidé. La experiencia de tutorías en la Universidad de Antioquia.

Memorias del Congreso Internacional.

Intercambio de experiencias en programas universitarios de Tutorías.

Universidad del Rosario. Bogatá. 2002

(3) GRUP DE TREBALL. Seminari permanent de tutoría universitaria, Institut de Ciencies de l' Educació. Unitat d' orientació universitaria. Institut de Ciencies de L'Educació. Lleida. España 2003

(4) BERNAL, Hernando. "El marco conceptual para el diseño de la Universidad". Revista de colegio Mayor de Nuestra Señora del Rosario 90:81$91,1997$.

(5) Plan Integral de Desarrollo 2004-2015. Crecimiento con calidad e identidad. Universidad del Rosario.

(6) Departamento de Admisiones. Guia de entrevista. Pregrados. Universidad del Rosario. 2003

(7) Documento de Investigación No 2. Línea de Investigación en Estado, políticas públicas y discapacidad. Reflexiones en torno a los conceptos de estado, las politicas públicas y la participación social. Facultad de Rehabilitación y Desarrollo Humano. 2004

(8) Documento politica del programa de tutoria. Universidad Colegio Mayor de Nuestra Señora del Rosario, Bogotá. Marzo 2000.

(9) PÉREZ, Luis. Universidad: Transformación o decadencia. Editorial Universidad de Antioquia. En: PINZÓN, de S; DIAZ, A. Excelencia académica y formación integral. Memorias del Congreso Internacional. Intercambio de experiencias en programas universitarios de Tutorias. Bogotá. Página 58. 2002.

(10)VÁSQUEZ, F. Proteger sin cortar las alas, riesgos y rasgos del maestro tutor. Facultad de Educación. Pontifica Universidad Javeriana. 2002
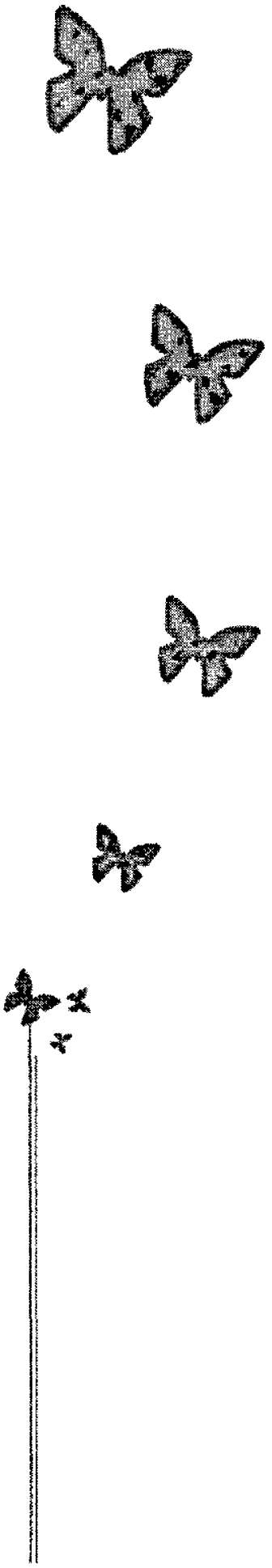